\title{
Interpolation of Body Surface Potential Maps
}

\author{
Bob J. A. Schijvenaars, MSc, * Jan A. Kors, PhD,* \\ Gerard van Herpen, MD, PhD, * Fred Kornreich, MD, PhD, + \\ and J. H. van Bemmel, PhD*
}

\begin{abstract}
The performance of four methods for interpolation of body surface potential maps (BSPMs) for different electrode grid densities was assessed. This study is part of a research project on the influence of the variability of 12-lead electrocardiograms on computer interpretation due to small electrode position changes. Interpolated BSPMs can be used to simulate this variability. The set of BSPMs studied, derived from a 117-electrode grid with relatively many electrodes on the left precordial part of the thorax, consisted of 232 cases without abnormalities, 277 with infarction, and 237 with left ventricular hypertrophy. The interpolation methods used were fast Fourier transforms, Chebyshev polynomials, linear functions, and cubic splines (CS). In the horizontal plane, a reference signal was first interpolated and, thereafter, resampled using 11 different sets of electrodes with the number of electrodes ranging from 18 down to 8 . In the vertical direction, five grids with electrodes only on the front of the thorax and nine grids with electrodes on the front and the back were examined. As a performance measure for interpolation, mean absolute error (MAE) was used: the absolute differences between the reference signal and the interpolated signal, averaged over the QRS on all maps. All methods showed deteriorating performance for decreasing grid density. In the horizontal direction, CS proved to be slightly superior to other methods for the left precordial electrodes for all but the densest grid (eg, $\mathrm{MAE}=22.8 \mu \mathrm{V}$ vs $\mathrm{MAE}>24.8 \mu \mathrm{V}$ for a 12 -electrode grid) For electrodes not in that area, CS performed the best as well ( $\mathrm{MAE}=16.1 \mu \mathrm{V}$ for the same grid), with differences with the other methods being small (MAE $>16.4 \mu \mathrm{V})$. In the vertical direction, CS showed the best results on the front, both for the dense nonperiodic (MAE $=19.1 \mu \mathrm{V}$ vs $\mathrm{MAE}>26.6 \mu \mathrm{V}$ for a 6 -electrode grid) and periodic grids ( $\mathrm{MAE}=25.1 \mu \mathrm{V}$ vs $\mathrm{MAE}>26.6 \mu \mathrm{V}$ for a 12 -electrode grid). Linear functions performed best for sparse nonperiodic grids and sparse periodic grids for electrodes on the back, with the difference with CS for the last case being small. The method CS performed best overall, and is recommended for interpolating BSPMs. Key words: body surface potential maps, electrode grids, fast Fourier transforms, Chebyshev polynomials, linear functions, cubic splines.
\end{abstract}

From the *Department of Medical Informatics, Erasmus University, Rotterdam, The Netherlands, and UUnit of Cardiovascular Research and Engineering, Free University of Brussels, Brussels, Belgium.

Reprint requests: Bob J. A. Schijvenaars, MSc, Department of Medical Informatics, Faculty of Medicine and Health Sciences, P.O. Box 1738,3000 DR Rotterdam, The Netherlands. 
The benefit of body surface potential maps (BSPMs) as a technique for obtaining more detailed electrocardiographic (ECG) information than is possible with standard ECG recordings has been shown in various studies in the past (see De Ambroggi et al. ${ }^{1}$ for an overview). The presentation of information is mostly in the form of isopotential surface maps, in which points of equal potential are joined to make isopotential contours. Recently, we have used BSPMs to study the effect of electrode position changes on ECG interpretation. ${ }^{2}$ In that study, electrode position changes were simulated by generating ECGs from BSPMs.

To obtain detailed isopotential surface maps, as well as to simulate electrode position changes, the number of measured locations needed is much larger than the number of electrodes that can be used in practice. Therefore, interpolation is necessary to achieve a detailed enough map. Research on which interpolation method to use has been done by Monro and Attwood ${ }^{3-5}$; however, the maps to be interpolated in these studies were relatively sparse ( 8 electrodes in both directions) and consisted of electrodes placed on an equidistant grid. Because a large fraction of the information is located near the middle of the thorax, where the heart is closest, a nonuniform electrode grid may therefore yield more information. Attwood and Monro analyzed several interpolation methods, both for horizontal and vertical interpolation, but quantitative results on the performance of these methods were given for only 10 BSPMs of mainly normal subjects and for 1 grid density. ${ }^{4}$ However, not all methods perform equally well for all grid densities, so the performance of the interpolation methods should be related to grid density.

For some interpolation methods, the signals to be interpolated are assumed to be periodic. In the horizontal direction, the signals are space-periodic, since traversal of a horizontal row ends where one started. In the vertical direction, this is not the case, and Monro introduced spatial periodicity by using two additional electrodes, one near the umbilicus and one on the neck. ${ }^{3}$ These two extra electrodes were used to introduce periodicity for all vertical rows. Because such electrode compositions can introduce undesired aliasing frequencies in the spatial signal, using an interpolation method that does not require periodic signals may be a better alternative for vertical interpolation. This has not been investigated in previous studies.

The purpose of our study was to assess the performance of several methods for interpolation of BSPMs for different grid densities. In this study, some of the limitations of previous studies were avoided. First, the grid we used was not equidistant in terms of electrode distance, and the BSPMs we used comprised 117 torso electrodes and extended to the umbilical level. Many electrodes were placed in the left precordial area, as can be seen in Figure 1 that shows a schematic unfolded thorax with the BSPM lead positions. Second, we used a set of 746 BSPMs from healthy subjects and patients with infarction or left ventricular hypertrophy. Third, the performances of the methods could be related to grid density since the methods were used to interpolate a number of different grids.

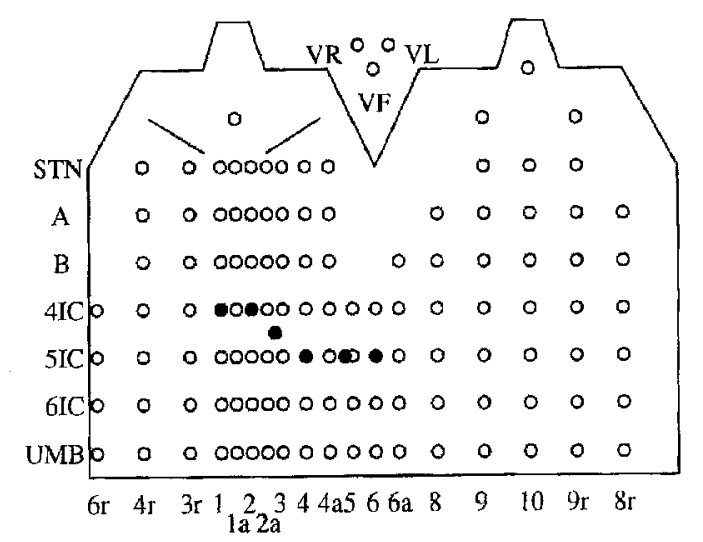

Fig. 1. An unfolded thorax showing the body surface potential mapping electrode positions. The left part of the image denotes the front and the right part denotes the back of the thorax. The electrode rows were placed $5 \mathrm{~cm}$ apart, with the fourth row from the bottom located on the fourth intercostal space $(41 \mathrm{C})$. The top row is at the level of the sternal notch (STN) and the bottom row is near the umbilical level (UMB). The horizontal placement was done using anatomic landmarks. The filled circles indicate the standard lead $V_{1}-V_{6}$ electrode positions. The three upper electrodes labeled VR, VL and VF denote the electrodes on the extremities.

\section{Materials and Methods}

\section{Body Surface Potential Maps}

A set of 746 BSPMs was used for the experiments. The procedure for recording the BSPMs has been described previously ${ }^{6,7}$ A BSPM consisted of 117 torso and 3 limb electrode sites. Figure 1 shows a schematic unfolded thorax with lead positions. The set of BSPMs consisted of $232(31 \%)$ cases without abnormalities (normal subjects), 277 (37\%) with infarction, and $237(32 \%)$ with left ventricular hypertrophy. These diagnoses were assessed on the basis of ECG-independent evidence. ${ }^{6}$ For each electrode position, a representative complex was obtained by coherent averaging.

\section{Interpolation Methods}

Four interpolation methods have been studied: interpolation using fast Fourier transforms, Chebyshev series interpolation, linear interpolation, and cubic spline interpolation. In the following, it is assumed that a signal $\mathrm{x}$ of length $\mathrm{N}$ (samples $\mathrm{x}_{0}$ to $\mathrm{x}_{\mathrm{N}-1}$ ) is interpolated to obtain a signal y of length $\mathrm{pN}$ (samples $\mathrm{y}_{0}$ to $\mathrm{y}_{\mathrm{pN}-1}$ ), with $\mathrm{p} \in \mathbb{N}$.

Fourier Interpolation. For Fourier interpolation, a signal is transformed using the discrete fast Fourier transform: zeroes are added in the middle of the transformed signal and this signal is then transformed back again to yield an interpolated signal (see Rabiner and Gold ${ }^{s}$ for a 
more elaborate discussion of the method). Let $X$ and $Y$ be the discrete fast Fourier transforms of $x$ and $y$ respectively. For $\mathrm{N}$ even, $\mathrm{Y}$ can be computed from $\mathrm{X}$ using:

$$
Y_{r}= \begin{cases}X_{r} & \text { for } r=0, \ldots, \frac{N}{2}-1 \\ X_{r} / 2 & \text { for } r=\frac{N}{2} \\ 0 & \text { for } r=\frac{N}{2}+1, \ldots, \frac{p N}{2}-1\end{cases}
$$

The second half of $Y_{r}$ (for $\left.r=p N / 2, \ldots, p N-1\right)$ is defined as the Hermitian symmetric of the first half. For $\mathrm{N}$ odd, the second right-hand side expression is not used. To obtain the interpolated $\mathrm{y}\left(\mathrm{y}_{0}\right.$ to $\left.\mathrm{y}_{\mathrm{pN}-1}\right)$, the inverse discrete fast Fourier transform is applied to $Y$.

Fourier interpolation is based on three assumptions. First, the sampling frequency should be sufficiently high to rule out aliasing. Second, the signal has to be periodic. Third, sampling must be equidistant. If one of these conditions is not met, the interpolation method will generate false harmonics.

Chebyshev Interpolation. Interpolation using Chebyshev series is closely related to Fourier interpolation. The method uses a least-squares polynomial fit. One of the definitions of the Chebyshev cosine series is (see Monro ${ }^{3}$ for more details):

$$
\begin{gathered}
C_{n}=\frac{2}{N} \sum_{j=0}^{N-1} x_{j} \cos \left\{\frac{(2 j+1) n \pi}{2 N}\right\} \\
n=0,1, \ldots N-1
\end{gathered}
$$

where $\mathrm{C}$ is an array of $\mathrm{N}$ real Chebyshev coefficients. The interpolated sample $\mathrm{y}_{\mathrm{k}}$ is:

$$
y_{k}=\frac{C_{0}}{2} \sum_{n=1}^{N-1} C_{n} \cos \left\{n \arccos \left(\frac{k}{p}\right)\right\}
$$

Another way of describing the method is by using Fourier series. ${ }^{3}$ Of the methods studied, only the Chebyshev and Fourier interpolations use the complete signal for interpolating between samples. Like Fourier interpolation, Chebyshev polynomials require uniform sampling. Periodicity is not required for Chebyshev polynomials. Interpolation using Chebyshev polynomials has some useful properties: it results in an interpolated signal with a small maximum error while oscillations are evenly distributed across the signal.

Linear Interpolation. Linear interpolation uses only "local" information to interpolate:

$$
y_{k}=A x_{j}+B x_{j+1} \text { for } p j \leq k<p(j+1)
$$

where

$$
A=\frac{p(j+1)-k}{p}, B=1-A
$$

Equations 4 and 5 show that the interpolation result between two samples $x_{j}$ and $x_{j+1}$ is independent of the values of other, nonadjacent samples.

Cubic Spline Interpolation. Spline interpolation is a method that divides a signal into sections that are separately interpolated using polynomials. In fact, linear interpolation is a form of spline interpolation using polynomials of degree 1 . In the case of cubic spline interpolation, the polynomials are cubic. The goal of cubic spline interpolation is to get an interpolated signal that is smooth in the first derivative and continuous in the second derivative, both within the interval and at its boundaries. Imposing these conditions on the coefficients of the interpolation polynomial between samples $\mathrm{x}_{\mathrm{j}}$ and $\mathrm{x}_{\mathrm{j}+1}$ results in:

$$
\begin{gathered}
y_{k}=A x_{j}+B x_{j+1}+C x_{j}^{\prime \prime}+D x_{j+1}^{\prime \prime} \\
\quad \text { for } p j \leq k \leq p(j+1)
\end{gathered}
$$

with $\mathrm{A}$ and $\mathrm{B}$ as in equation 5 and $\mathrm{x}$ " is the second derivative. Further,

$$
\begin{aligned}
& C=\frac{p^{2}}{6}\left(A^{3}-A\right) \\
& D=\frac{p^{2}}{6}\left(B^{3}-B\right)
\end{aligned}
$$

Requiring continuity of the second derivative across interval boundaries gives $\mathrm{N}-2$ equations in the $\mathrm{N}$ unknown $x^{\prime \prime}{ }^{\prime}$ for $j=0, \ldots, N-1$. To solve for the $N$ unknowns, two more equations are required. The two equations are often specified as preset values for $\mathrm{x}_{0}^{\prime}$ and $\mathrm{x}_{\mathrm{N}-1}^{\prime}$ or zero values for $\mathrm{x}^{\prime \prime}{ }_{0}$ and $\mathrm{x}_{\mathrm{N}-1}$. When the signal is assumed to be periodic, $\mathrm{N}$ equations result because $\mathrm{x}^{\prime \prime}{ }_{0}$ and $\mathrm{x}_{\mathrm{N}-1}$ can then be computed in the same way as the other second derivatives $\mathrm{using} \mathrm{x}_{\mathrm{j}}=\mathrm{x}_{\mathrm{j} \bmod \mathrm{N}}$

\section{Experiments}

The performance of the different interpolation methods was assessed separately tor the vertical and horizontal directions. For the horizontal direction, the row at the level of the fifth intercostal space at lead $V_{4}$ was used (row 51C in Fig. 1). For the vertical direction, one column on the front of the thorax and one on the back (columns 4 and $8 \mathrm{r}$ in Fig. 1) were used. Figure 2 shows a schematic of the experimental setup. Based on the original samples in the row or column (indicated by the top array in Fig. 2), a set of densely spaced samples were derived, to be used as the underlying reference signal $r$ (array 2). We assumed that the original samples were an adequate representation of the underlying signal, so that the Fourier approach could be used to produce the underlying signal by exact interpolation. Different grid densities were then simulated by taking equidistant samples from this reference signal (array 3). The density of the densest grid thus constructed was equal to the density of the original grid. This signal was interpolated again 


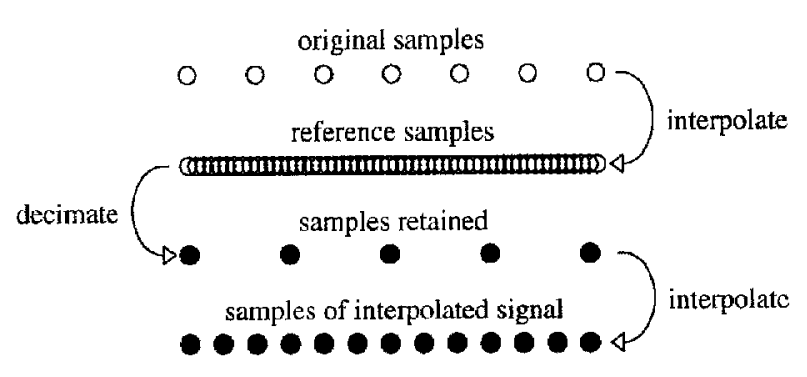

Fig. 2. Experimental setup. The top array indicates the original samples in the body surface potential map. Interpolation of this original signal yields underlying reference samples (array 2). This reference signal is resampled by retaining a small subset of samples. The signal thus produced (array 3 ) is submitted to the four interpolation methods. The samples of the interpolated signal (array 4) are then compared to the corresponding samples in the reference signal (array 2).

by the different methods studied. The resulting interpolated signal s (array 4) was then compared to the reference signal $r$.

The performance of each interpolation method was assessed by computing the mean absolute error (MAE) between the reference signal $r$ and its interpolated counterpart $s$ over the time-normalized QRS complex and over all BSPMs:

$$
\text { MAE }=\frac{1}{M N} \sum_{n=1}^{N} \sum_{m=1}^{M}\left|s_{n, m}-r_{n, m}\right|
$$

where $M$ is the number of BSPMs used $(M=746)$ and $N$ is the number of time instances in the time-normalized QRS complex ( $\mathrm{N}=35)$. The MAE, like the root-meansquare error, is a global indicator for the similarity between two signals and has been chosen for the sake of comparability; Attwood and Monro used the same measurement in their experiments. ${ }^{4}$

For each grid density, the reference signal was resampled several times with different phase, that is, with a slightly different offset. The resulting MAE values were averaged, thus increasing the accuracy of the MAE values.

Horizontal Experiments. In the horizontal experiments, the reference signal was based on the samples from the 18 electrodes in row 5IC (Fig. 1). The position of these electrodes relative to each other is indicated in a cross-section of the thorax in Figure 3. The reference signal was obtained by interpolating this signal using fast Fourier transform, since the signal was space-periodic.

The sizes of the grids studied ranged from 18 down to 8 samples. These grids will be referred to as $\mathrm{Hl} 8, \mathrm{Hl} 7$, etc. The MAE values were computed for two subsets of the reference locations. The first subset consisted of the reference locations from column 1 to 6 , that is, encompassing the left-precordial area. The other subset consisted of all other reference locations.

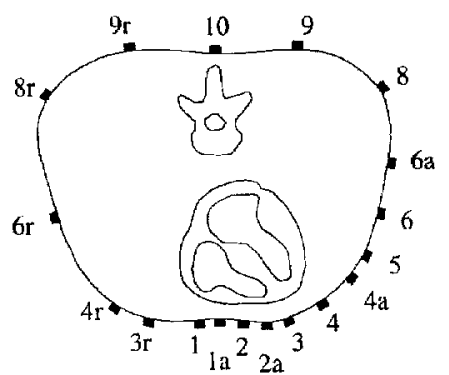

Fig. 3. Horizontal cross-section of the thorax showing the approximate locations of the electrode columns with respect to the heart center and the thorax center. The column names correspond with those in Figure 1.

Vertical Experiments. In the vertical experiments, three reference signals were used: one periodic and two nonperiodic. The periodic reference signal was generated using the 16 electrode samples. In addition to the electrodes in columns 4 and 8 r, the top electrodes in columns $9 \mathrm{r}, 1 \mathrm{a}$, and 10 were used. The interpolation method used to generate the reference signal was fast Fourier transform.

The sizes of the periodic grids studied ranged from 16 down to 8 samples (referred to as VP16, VP15, etc.). The MAE values were averaged over two subsets of the reference locations. One subset (encompassing the front thorax) comprised the locations from the sternal notch to the umbilical level, the other one consisted of all reference locations in column $8 \mathrm{r}$ (A to the umbilical level).

The two nonperiodic reference signals were obtained by interpolation using the cubic spline method instead of fast Fourier transform since the latter method assumes a periodic signal. The first nomperiodic reference signal was based on the samples in column 4 and on the top electrode in column la. The other nonperiodic reference signal was based on the samples on the back of the thorax; all samples in column $8 \mathrm{r}$ and the top electrodes of columns $9 \mathrm{r}$ and 10 .

The nonperiodic grids had sizes ranging from eight down to four samples (VN8, VN7, etc.). The MAE values were averaged over the same subsets as in the periodic vertical experiment.

\section{Results}

\section{Horizontal Experiments}

Figure $4 a, b$ shows that the performance of all interpolation methods decreased with grid density. The linear functions method performed worst on the left-precordial area for all grid densities. The fast Fourier transform and Chebyshev polynomials methods (the results of which were almost identical) performed worst on the back for most grids. Even though the reference signal was obtained by interpolation using fast Fourier transform, the cubic spline method performed best for all grids except $\mathrm{HI} 7$ and $\mathrm{Hl} 8$. 

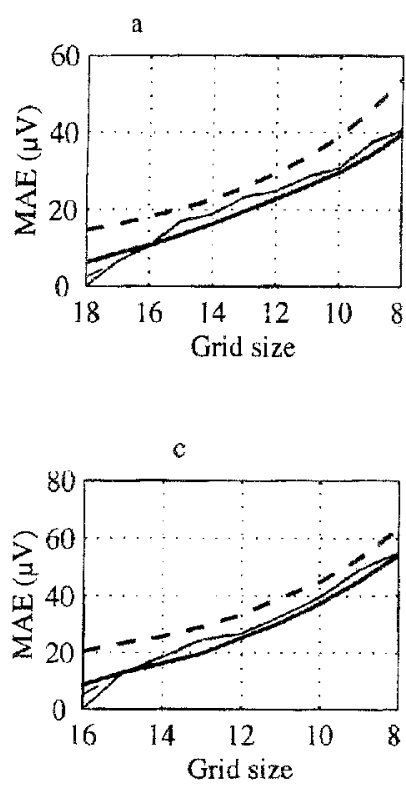

$\mathrm{e}$

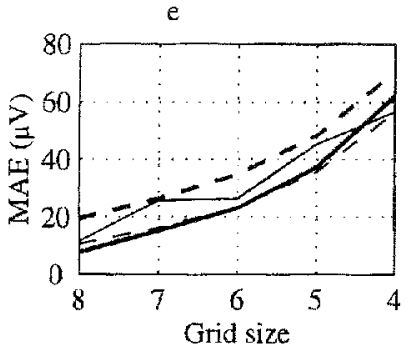

$b$

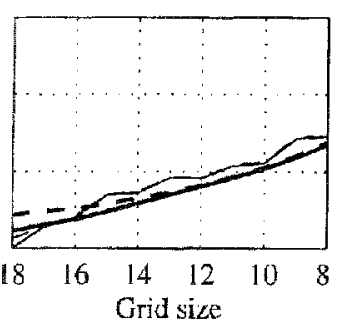

d
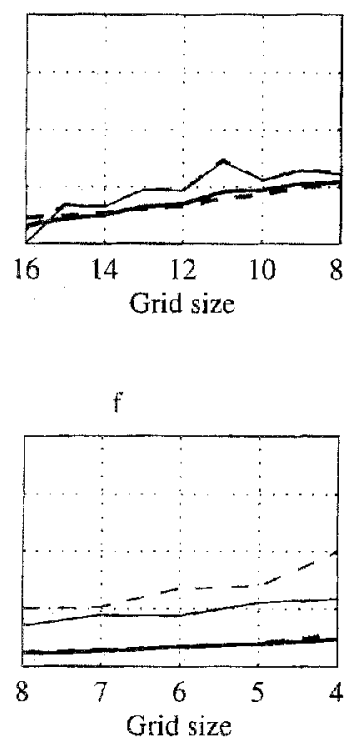

Fig 4. Mean absolute error (MAE) values of the different experiments as a function of grid size. (a) Horizontal interpolation, results for the electrodes in the left precordial region. (b) Horizontal interpolation, results for the other electrodes (not in the left precordial region). (c) Vertical interpolation of periodic signals, results for electrodes on the front of the thorax. (d) Vertical interpolation of periodic signals, results for electrodes on the back of the thorax. (e) Vertical interpolation of the nonperiodic reference signal, results for electrodes on the front of the thorax. (f) Vertical interpolation of nonperiodic signals, results for electrodes on the back of the thorax. Thin lines denote the results of fast Fourier transform (solid line) and Chebyshev polynomials (dashed line). Thick lines denote the results of cubic spline interpolation (solid) and linear functions (dashed). If only one thick line is shown, fast Fourier transform and Chebyshev polynomials coincide.

\section{Vertical Experiments}

The results for the periodic vertical experiments are shown in Figure 4c, d. The linear functions method performed worst for the electrodes on the front of the thorax, but performed well on the back, where the fast Fourier transform and Chebyshev polynomials methods performed worst. Cubic spline interpolation performed relatively well for both areas.

Figure $4 \mathrm{e}$, f shows the performances of the interpolation methods for the nonperiodic vertical grids. Note that only in these experiments was the reference signal obtained by interpolation using the cubic spline method. The performance of the Chebyshev polynomials method was relatively good on the front of the thorax but worst on the back. Fast Fourier transform probably suffered from the fact that its condition of a periodic signal was not met. Cubic spline interpolation performed best for most grids. These results are difficult to compare with those of the periodic vertical experiments because different reference signals were used.

The results of the experiments show a superior performance for fast Fourier transform and Chebyshev polynomials for the densest horizontal grids (H18 and H17) only. In most other grids, cubic spline interpolation performed better for the three experiments.

\section{Discussion}

The results suggested that the best overall interpolation method for electrode grids of different densities was cubic spline interpolation. Only for the densest periodic grids was its performance surpassed by that of the fast Fourier transform and Chebyshev methods. For fast Fourier transform, the MAE increase when going from grid VP15 to VPI4 was much less than the MAE increase going from VP16 to VP15. This suggests a relatively high contribution to the signal power of the highest frequency sampled in the reference signal. That may have been due to disturbances generated by large differences between the electrodes at the umbilical level of columns 4 and $8 \mathrm{r}$. This is supported by the fact that the difference between electrodes 6IC and UMB on column la (cf. Fig. 1) averaged over all maps is lower than the averaged difference between electrodes UMB on columns 1a and 10: a "jump" in the signal occurs. If so, then connecting columns to introduce periodicity will not improve accuracy.

For horizontal interpolation, Attwood and Monro reported MAE values averaged over 10 patients of 75.5 , 48.6, and 54.1 $\mu \mathrm{V}$ for the fast Fourier transform, Chebyshev polynomials, and cubic spline methods, respectively, for a grid of eight electrodes. ${ }^{4}$ Corresponding values in this study can be obtained by averaging the MAE values of grid H8 over all reference electrodes. The resulting values are $34.3,34.5$, and $32.7 \mu \mathrm{V}$, respectively. There is a striking difference between these two sets of values, which may be caused by a difference in the pattern of electrode locations. Attwood and Monro's horizontal grid was equidistant, meaning there were as many electrodes on the front of the thorax as on the back. The horizontal grids used in this study were not equidistant, since relatively many electrodes were located on the front of the thorax (cf. Figs. 1, 3).

For the vertical periodic grid, Attwood reported MAE values of $44.0,42.6$, and $35.3 \mu \mathrm{V}$ for the fast Fourier transform, Chebyshev polynomials, and cubic spline methods, respectively. Corresponding errors resulting from averaging the MAE values of grid VP8 over all refer- 
ence electrodes were $47.2,47.6$, and $40.9 \mu \mathrm{V}$. In this case, our results are slightly higher than those of Attwood.

It is difficult to assess which interpolation method is best for interpolation of even denser sampled BSPMs. In the horizontal direction, Fourier interpolation is theoretically preferred if the sampling frequency is high enough and the spatial signal not aliased. Antialias filtering of the spatial signal is not possible, however. Cubic spline interpolation performed best in most other grids and does not impose any requirements, so it might be an alternative. The differences between the performance of the methods for such dense grids will be small, however.

The question of how many electrodes to use for interpolating a BSPM depends on the accuracy desired. Typical root-mean-square noise values in the precordial leads are less than $15 \mu \mathrm{V} .^{10}$ This corresponds with an MAE value of about $20 \mu \mathrm{V}$ for noise with a Gaussian distribution. For interpolation accuracy higher than this value, the horizontal grid should consist of more than 13 electrodes. In the vertical direction, 14 electrodes should suffice ( 7 on the front of the thorax and 7 on the back). Cubic spline interpolation is recommended. It should be noted that the performance of the interpolation methods was assessed using a quantitative measure of signal differences; however, the relationship between signal differences and changes in diagnostic interpretation is not straightforward. Thus, the accuracy of the methods studied may not faithfully represent their ability in preserving diagnostic information.

\section{References}

1. De Ambroggi L, Musso E, Taccardi B: Body-surface mapping. p. 1015. In Macfarlane PW, Lawrie TDV (eds): Comprehensive electrocardiology. Pergamon Press, Oxford, 1989

2. Schijvenaars RJA, Kors JA, Van Herpen $G$ et al: The effect of electrode position changes on ECG interpretation by computer. Internal report, Department of Medical Informatics, Erasmus University, Rotterdam, October 1994

3. Monro DM: Interpolation methods for surface mapping. Comput Program Biomed 11:145, 1980

4. Attwood RD, Monro DM: Reconstruction methods for body surface maps from 26 leads. p. 463. In Macfarlane PW (ed): Progress in electrocardiology. Pittman Medical Press, 1979

5. Monro DM, Guardo RAL, Bourdillon PJ, Tinker J: A Fourier technique for simultaneous electrocardiographic surface mapping. Cardiovasc Res 8:688, 1974

6. Kornreich F, Montague TJ, Rautaharju PM: Body surface potential mapping of ST segment changes in acute myocardial infarction. Circulation 87:773, 1993

7. Montague TJ, Smith ER, Cameron DA et al: Isointegral analysis of body surface maps: surface distribution and temporal variability in normal subjects. Circulation 63:1166, 1981

8. Rabiner LR, Gold B: Theory and application of digital signal processing. Prentice-Hall, Englewood Cliffs, 1975

9. Press WH, Teukolsky SA, Vetterling WT, Flannery BP: Numerical recipes in C: the art of scientific computing. 2nd ed. Cambridge University Press, Cambridge, 1992

10. Kors JA, Van Herpen G, Van Bemmel JH: Variability in ECG computer interpretations: analysis of individual complexes vs analysis of a representative complex. J Electrocardiol 25:263, 1992 